venous bleeding from the tumor mass. When you have sutured over the stump you have reformed the convex pelvic roof instead of making a concave one, and a minimum amount of suturing is required. There is no loss of blood, no shock, and the patient is as free from all postoperative trouble as after a simple ovariotomy.

Dr. Chester M. Echols, Milwaukee: In our enthusiasm to cut something out, especially if it be a tumor which might become malignant, I think we are apt to overlook the definite indications for myomectomy as opposed to hysterectomy or supravaginal amputation. I admit that the indications are not present in the majority of cases, but in young married women there should be left undisturbed the function of childbearing and of menstruation if at all possible. In my student days I was taught that myomectomy was more dangerous than hysterectomy because of the possibility of bleeding and other complications. My experience in a considerable number of myomectomies has convinced me that its indications are often overlooked. One case illustrates quite a class: Two and a half years ago the wife of a prominent physician in our city was desirous of having children. Because of the presence of multiple fibroids of moderate size she was advised to have a hysterectomy. I examined her and advised that she have a myomectomy done, which she did. She is now the happy mother of a three or four months old baby. It is interesting to note that careful examination shows that there has been no recurrence of tumors, not even to the size of a cherry. Hysterectomy can always be done as a last resort in case of recurrence of the tumors, and it is an operation practically devoid of danger.

Dr. J. A. Ruben, Pittsburgh: If the use of Roentgen rays. and radium in the treatment of malignant diseases or tumors of the uterus can be considered malpractice, then the use of any new remedy, not universally accepted, must be considered malpractice. Regarding the old idea of Roentgen rays causing cancer, it may be said that the earlier roentgenologists who sacrificed their lives trying to develop something new that would relieve or cure disease did so in ignorance of the physical character of the force to which they exposed themselves and their patients. They did not know that the Roentgen rays and the rays of radium could be divided into soft, medium and hard rays. The soft rays. have so little penetrating power that they can be stopped by a sheet of paper, and are mostly responsible for the burns of both patients and operators. On the other hand. the hard rays have such power of penetration that they may pass through armor plate; these cause little or no injury to normal tissues, even in very large doses. Of the pioneers, in this work, probably some will die of cancer-some havebut today we understand better what we are handling. With the use of the radiometer for measuring the Roentgen ray doses and with standardized technic ( 3 millimeter thickness of aluminum or lead as filter). even in the treatment of deep seated conditions, our patients suffer no harm. The operator also, with modern protection, standing behind a leaded wall, can feel perfectly safe. Finally, a number of gynecologists are now doing this work themselves, and under their own careful observation, can soon distinguish those cases unsuitable for radiotherapy, and in sincere honesty, advise a timely operation.

Dr. Stephen E. Tracy, Philadelphia: I do not believe that all fibromyomata uteri should be treated by surgery: nor do I believe all should be treated by roentgenotherapy: I question the sincerity of the man who claims he can cure all such tumors. except those removable by vaginal myomectomy, by roentgenotherapy. I agree with Dr. Schmitz that some cases should have roentgenotherapy. Dr. Pfahler will not treat a case of fibromyomata uteri unless referred to him by a surgeon; he wants to know what he is treating. In fibromyonata uteri associated with malignancy, I believe that early radical operation will give the best results. Formerly, Roentgen treatment for malignant disease in the female pelvic organs was practically useless. Now, roentgenologists claim that with a better technic, more powerful machines, anci a competent operator, much may be accom- plished in these cases. In view of these claims, it would seem that better results should be secured in cases of fibromyomata uteri associated with malignancy if the radical operation be followed by systematic Roentgen treatment by a competent operator, the same as is done in carcinoma of the breast and in other superficial malignancies. I have never seen a case of fibromyomata uteri in which I was afraid to operate, and my mortality does not indicate that I have been reckless. All my patients have been studied carefully, and in some cases it has taken weeks, and in a few even months to get the patient in a sufficiently good conidition to undergo an operation with a reasonable hope of a satisfactory result. I agree with Dr. Carstens that material removed by the curet should be examined microscopically Dr. Lawrence referred to his method of hysterectomy. This will no doubt work out well in some cases, but to use such a technic, it is necessary to have cases made to order. His technic cannot be carried out in large intraligamentous nodules, or in tumors associated with inflammatory lesions. I fully agree that the cervical stump should be sutured to the infundibulopelvic and round ligaments. This we always do. but with a different technic. Dr. Echols spoke of myomectomy. In the paper I stated that we should know the age at which the degenerations take place in these tumors in order to decide whether we should do conservative of radical surgery. In women under 40 , if possible, a myomectomy should be done, after 40 , as a general rule, on account of the changes in the uterus and tumor, a hysterectomy is the better operation.

\section{BACTERIAL VACCINES IN TREATMENT OF PULMONARY TUBERCULOSIS}

\section{IMPRESSIONS AS TO CLINICAL VALUE *}

\section{S. G. BONNEY, M.D. DENVER}

Although I have had opportunity to note the apparent effect of vaccine therapy, independently of tuberculin, in a large number of cases, I will submit merely a preliminary statement of general impressions derived from clinical observation:

Do bacterial vaccines exert an influence for good in pulmonary tuberculosis, and, if so, under what conditions and with what restrictions?

Are they capable of doing harm, and if so, what pre cautions should be observed in, and what are the special contraindications for their use?

My position, from the data thus far obtained, is that 1. Vaccine therapy should by no means be permitted as a routine measure in the treatment of tuberculosis.

2. It has a legitimate place as a tentative procedure in a class of cases subject to certain modifying limitations.

3. Careful adjustment of dosage is vitally important.

4. The number of pulmonary invalids for whom vaccines are properly applicable is comparatively small.

5. The results obtained are very uncertain.

6. The proportion of those exhibiting improvement is disappointing.

7. The gain established in a few is sometimes impressive.

8. Vaccines not infrequently are shown to possess rast possibilities of injury.

9. Finally, the attitude of the profession should be one of the utmost conservatism.

It may be added parenthetically that the foregoing statements seem to be true, to a certain extent at least, of tuberculin therapy.

* Read before the American Climatological Association at the Annual Meeting, Washington, D. C., May 10, 1916. 
Apropos of these rather definite opinions, attention should be called to the not infrequent delusion regarding the efficacy of this treatment, to the indiscriminate administration of vaccines for nearly all classes and conditions of pulmonary tuberculosis, and to the deplorable results occasionally noted from the abuse of an agent which, intelligently employed, may become at times of genuine value.

A single case may serve as an illustration of the careless empiricism displayed in the use of bacterial vaccines.

CASE 1.-A woman, aged 32, came under my observation in June, 1915, presenting the history of a tuberculous infection seven years previously. Since that time she had visited various health resorts and sojourned in several sanatoriums, submitting to tuberculin medication for prolonged periods, as well as to the administration of bacterial vaccines, with subsequent unfavorable results. She came to Colorado two weeks after a rather severe hemorrhage, exhibiting loss of weight, very considerable elevation of temperature, dyspnea and occasional chills and night sweats. There were physical evidences of active tuberculous involvement in the right lung from the apex to the third rib and to the lower edge of the shoulder blade. There was also active involvement in the left lung. Tubercle bacilli were numerous in the sputum. For months the tendency had been that of a progressive decline. In the face of these clinical manifestations and physical signs, the patient was being given, at intervals of from one to three days, a stock vaccine containing six different micro-organisms. No culture had been taken of the sputum and hence no information had been obtained as to the nature of the infection, yet the patient was provided with an elaborate outfit and directed to have her physician or nurse administer the vaccine in accordance with instructions received.

As there was no clinical or bacteriologic basis affording the slightest justification for this treatment, it was promptly suspended, and the patient was committed to a systematic regimen in accordance with conservative methods. In ninety days the fever was under complete control and there were other evidences of marked clinical improvement, including a gain in weight of 15 pounds and a pronounced lessening in the activity of the tuberculous process.

The development of unfavorable symptoms following the use of bacterial vaccine should always demand its prompt discontinuance.

In this connection it is interesting to note a similar illustrative example of the misuse of tuberculin:

Case 2.-In May, 1915, a patient from one of the large Eastern cities presented a letter of introduction from an active practitioner who described the condition, reported the use of an autogenous vaccine and directed that at regular intervals $3.5 \mathrm{mg}$. of Koch's old tuberculin be given.

Examination of the chest disclosed the presence of an active and extensive tuberculous involvement in the left lung, moist râles being recognized from apex to base. The sputum was literally peppered with tubercle bacilli. It is almost unnecessary to state that the tuberculin was discontinued and the patient put in bed. The temperature has since been normal, he has gained 35 pounds in weight, the cough and expectoration have entirely disappeared, and physical examination reveals but an occasional semidry click just outside the left precordial region.

Can any one fail to recognize the relation of cause and effect in such instances as these?

It is possible that the popularity of bacterial vaccines as a conventional measure for pulmonary disease has not been as frequently observed by other clinicians in health resorts. In this event, my impression as to their widespread employment before resorting to climatic change must be regarded as one of the anoma- lies of my experience. While the detrimental effects of injudicious specific medication are not always so conspicuous as in the cases cited above, evidences of an unfavorable influence are not wanting in my own cases, as shown by occasional chills, elevation of temperature, increase of cough, malaise, or varying degrees of prostration. While these disturbing manifestations may of course be attributed to improper dosage and, therefore, are capable of later amelioration or suppression, a happy adjustment of size and frequency of injection is not always to be attained. In the absence of precise laboratory methods to control their use, the results of their administration, especially under present methods of preparation, inevitably become a matter of extreme uncertainty. As usually prepared, even by those well equipped for this work, there is no positive assurance that the micro-organisms constituting the vaccine are identical with those chiefly responsible for the infection and its consequences in the individual. Cultures taken on successive days with equal care do not always furnish the same results. The use of vaccines representing the isolation and incorporation of several varieties of micro-organisms is necessarily subject to still greater hazards. Notwithstanding accurate standardization, the clinician in such instances possesses no means by which to adjust separately the dosage of the several component ingredients. While a degree of immunity may thus be established for one infection, no corresponding influence is exerted on the others. It is entirely possible that, owing to modifying influences pertaining to the host and to important changes in the invaders incident to environment and adaptation, certain constituents of the vaccine may later become not only useless but actually harmful by prolonging the negative phase and lessening individual resistance.

It would appear that the use of several separate vaccines, each consisting of but one type of microorganism, would only add to the confusion, because of the difficulty of such an interspersing of doses, in allowing opportunity for clinical deductions.

In these cases the work with the complement fixation test is of interest and worthy of commendation. It is perfectly conceivable that in some instances the information obtained by this means may be of the utmost value. This has been well shown recently in two of my cases, in which the patients exhibited an obscure fever with other symptoms suggesting tuberculosis, which caused their removal to Colorado. In both instances the infection was shown by the complement fixation test to be gonococcic in nature and was favorably influenced by vaccine therapy. Some difficulties, however, are at present encountered in employing this test to ascertain the predominant secondary infection in pulmonary tuberculosis.

It should perhaps be recognized that embarrassment may result from the existence of intercurrent complications. An influenza or other infection, perhaps of remote origin, may yield a positive response on exhibition of the complement fixation test and yet be without especial significance as regards the lessened individual resistance occasioned by another secondary infection. Thus in some cases it may be impossible to conclude that a positive test from one organism affords any direct information as to the infection from which the patient is actually suffering. Furthermore, even in the presence of a positive test, the lack of definite control renders it impossible to measure with any degree of accuracy the extent of the immunity established. 
As illustrative of some of these considerations the following case is of some interest:

CASE 3.-In March, 1913, a patient came to Colorado fifteen months after the development of initial pulmonary symptoms. There was great loss of weight, prostration, fever, dyspnea, cough and expectoration, with well defined physical signs in each lung, and especially active involvement of the left. As a result of rest in bed and other rational measures and, without recourse to specific medication, a gain of 36 pounds resulted in the course of eight or ten months, with corresponding change for the better in all the clinical manifestations, including the physical signs. The improvement finally ceased, and was followed by a stationary period for several months, at the end of which a vaccine, prepared in ampules by Drs. Simon and Burdick, appeared to be of very substantial benefit in diminishing the cough and expectoration and lessening the moisture in the left chest. The vaccine was exhausted at the end of about three months, and a culture taken at that time disclosed an entirely different type of infection. Another vaccine was prepared by Dr. Burdick and, although administered for two or three months in steadily decreasing doses, exerted an apparent unfavorable influence, whereupon its use was suspended. After several months without appreciable change in the general condition of the patient, culture of the sputum revealed a still different combination of infections, and a vaccine was prepared by Dr. Waring. This seemed at first to have a beneficial effect, but later became apparently inert.

It is possible that in such an instance the information to be obtained from the use of the complement fixation test might prove of definite value. It is also suggested by this and other experiences that the effects produced by bacterial vaccines may be merely relative and the improvement but transitory, even though for a time an apparent increase in the defensive resources is developed. Desirable as it is to avoid the production of a reaction in such cases, an indication of tolerance is not necessarily an evidence of acquired immunity and is quite insufficient to justify an assumption as to the benefits afforded. Similarly, the absence of unpleasant symptoms does not always suggest the wisdom of continued increase of dosage, but the nature of the clinical manifestations may represent important contraindications.

In this connection especial attention should be devoted to the condition of the kidneys. In many instances these organs are already overtaxed in the effort to eliminate toxins incident to the tuberculous and mixed infections, especially those of streptococcic origin. An additional burden is often imposed by an injudicious attempt to increase largely a protein diet. Under a prolonged continuance of these conditions the administration of vaccines in large doses may be responsible for urinary findings indicative of further injury to the renal tissue.

Amid the obscurity involving many features of vaccine therapy and the inherent difficulties owing to the possible complicating conditions in pulmonary tuberculosis, arbitrary conclusions seem unwarranted. Provisional opinions are permissible only when possibilities of error are eliminated to the fullest extent. When the employment of bacterial vaccines represents simply the introduction of a new element in the therapentic regimen, all other conditions remaining approximately the same as before its use, it may be fair to assume a causal relation. Thus, sources of confusion, and doubt as to the value of this or any other medicinal agent must obtain, if administered to invalids shortly after radical climatic change, the physiologic influence of which may overshadow the effect of specific medication.
In like manner incorrect deductions are unavoidable, in the event of a coincident change of management or mode of life. In order to minimize the uncertainties of clinical opinion as to their value, it is essential that vaccines be used only in carefully selected cases, preferably those conforming to a rigid regimen for a considerable period without change of climate or environment. The conclusions thus afforded are made still more reliable if the application of this agent is limited to patients whose condition has become more or less stationary with the ultimate prognosis somewhat doubtful. In the absence of a definite progressive tendency in either direction, the development of a substantial clinical change following its use cannot be entirely disregarded. There exists no valid excuse for giving vaccines to patients who are doing well, and but little encouragement for a proper interpretation of results if given to those who are rapidly declining. While vaccines would seem especially appropriate for those exhibiting unsatisfactory progress, yet not entirely hopeless, it is not always possible, even in these cases, to attribute an improvement solely to the specific medication.

In addition to the remedial agent two factors may contribute quite materially to this result, the psychic element, which is worthy of practical consideration among pulmonary invalids, and the opportunity presented for closer observation and more careful direction. Even the fact of a demonstrable gain following the use of bacterial vaccines affords no actual criterion in all cases by which to estimate its specific medicinal effect. In the midst of the discouragement and pessimism engendered by periods of deferred hope, the moral uplift from the thought that something is being done is sometimes quite remarkable. An improved mental attitude, a better appetite and digestion and an increasing spirit of cooperation ensues. The more frequent contact of physician and patient, the influence of which is far-reaching, results in mutual sympathetic relations productive of earnest, interested effort on the part of each. Faithful compliance with instructions is thus secured and a correspondingly greater medical efficiency. Caution should be exercised, however, lest following the initial enthusiasm, the physician permit himself, in the frequent routine of vaccine administration, to devote insufficient attention to important details of management which constitute the very essence of any success to be obtained. The tendency under such circumstances to drift unconsciously from the recognized features of personal control may be quite pronounced. If this is carefully avoided, and due advantage taken of the facilities afforded for more detailed supervision, it is quite conceivable that distinct benefit may accrue from vaccine therapy, quite irrespective of its specific action.

The cautious employment of bacterial vaccines, as previously stated, would seem to be appropriate chiefly for persons who failed to show satisfying improvement under conservative management. I have restricted its use largely to this class of patients, as I could not consistently subject an invalid presenting every reasonable assurance of eventual recovery to the active influence of a therapeutic agent of uncertain value. It does not follow, however, that vaccines are indicated for all patients who are doing poorly. Such a broad classification would include not only those who, on account of the advanced infection, are clearly doomed to a fatal termination, but also others whose retardation of progress may be ascribed to important, though not 
hopeless complications, or to the development of disturbing conditions capable of amelioration under resourceful management. In many of these cases the unfavorable factors are so apparent as to suggest change of environment, removal of adverse influence, a more generous and appetizing cuisine, added opportunity for physical and mental repose and closer attention to other details, rather than to an abrupt recourse to a doubtful form of medication. In the absence of other pathologic conditions, in themselves explanatory of unsatisfactory general progress, and demanding special remedial measures, it may be granted that a demonstrated inability to respond favorably to an intelligent regimen suggests the expediency of specific therapy. In my earlier experience with vaccines, their application was largely confined to far advanced patients exhibiting a relentless decline with pronounced septic manifestations. All of these, by virtue of every consideration ordinarily influencing prognosis, were regarded as hopeless. In many of the cases, the course of the disease was uninfluenced, but in a few, definite improvement was noted, while in one or two conspicuous instances the clinical picture was unexpectedly transformed. On the whole, the results obtained in this class of cases have been extremely disappointing, and the impression has been received that but little if any encouragement is afforded from vaccine therapy under such conditions.

It would seem that the elaboration of protective substances within the individual from the introduction of a bacterial agent is most unlikely when, in approaching terminal states, the natural powers of defense have become exhausted. It is altogether probable that at such a time the machinery of immunization is inoperative because of the flooding of the blood with bacterial products without capacity for resulting formation of antibodies. When therapeutic stimulation of the active protective processes is thus impossible, an attempt toward the development of passive immunization by the injection of antibodies contained in the serum of immunized animals is justifiable, although likely to be of little avail owing to the difficulties of proper serum identification.

Notwithstanding the very proper objection to the rationale of this procedure, the fact remains that clinical experience has furnished evidence concerning its value in rather a small proportion of cases.

The fever of pulmonary tuberculosis, exclusive of the previously mentioned septic infections in advanced cases, may represent, more than any other feature of the disease, a possible indication for vaccine therapy. A persisting elevation of temperature, independent of the nature and extent of pathologic change, represents an insuperable obstacle to recovery, and hence the application of therapeutic measures for its control is especially significant. It is necessary, however, to discriminate with the utmost care between the fever of mixed infection and that dependent on other causes. It is, of course, absurd to expect benefits from bacterial vaccines when the fever is due to such unrecognized conditions as a pleural effusion, malaria or miliary tuberculosis. Instances of this kind are by no means infrequent, as well as obscure gallbladder infections, chronic appendiceal involvement, sinus disease, cystitis and colon infection of the kidneys. A recent experience has been particularly instructive in that the fever of a young man, shortly after arrival in Colorado, with pulmonary lesions, was found to be produced by paratyphoid B. infection. This information was acquired only after blood cultures, several Widal tests and an examination of the spinal fluid had elicited negative results, and the nature of the clinical manifestations had suggested a reasonable doubt as to the possible causal relation of a mixed infection, despite the detection of the secondary micro-organism in the sputum.

Elevation of temperature is not necessarily attributable to the accompanying bacteria of mixed infection. That absorption of the toxins of the tubercle bacillus may be responsible for fever in some cases is generally accepted.

While the secondary bacteria of mixed infection may play an important rôle in the development of the pathologic processes common to tuberculosis, and exert an influence on the clinical characteristics of the disease, similar constitutional manifestations may exist without recognition, after repeated examinations, of the specific micro-organism in the sputum or blood. Even the detection of the accompanying bacteria in the sputum is not of itself sufficient to confirm an assumption that the mixed infection is the sole responsible cause for the clinical symptoms. Their presence at least affords no reliable measure of the severity of the infection and the relative gravity of the situation, which for practical purposes is best estimated by the extent of temperature elevation. The expulsion of bacteria with the tuberculous sputum, in the absence of unfavorable clinical manifestations, is no indication for the employment of bacterial vaccines. Even when apparently characteristic eridences of mixed infection are present, an element of real doubt as to the propriety of vaccine therapy is unavoidable as the syndrome may, after all, be of essentially tuberculous origin. Irrespective of these considerations as to pathogenesis, and with a just appreciation of the inevitable uncertainties of action, it appears, nevertheless, permissible to resort to vaccine therapy under the conditions enumerated.

In my experience, the results obtained in a fair proportion of cases in which there was moderate fever have been such as to encourage its further cautious employment. No assurances as to its probable effect can be given, and patients should be made to understand that its use is largely experimental. Under a wisely directed management they have but little to lose from its trial and perhaps something to gain.

This is also true to a large extent in connection with its employment in afebrile cases. While the greater proportion of such invalids may be expected to make fairly satisfactory progress under proper supervision without recourse to vaccine therapy, occasional indications for its administration may be found in patients with distressing cough and abundant expectoration. Many of these cases without fever exhibit more or less cavity formation or bronchiectasis with varying degres of fibrosis. Under these conditions, even if but sight loss of weight has taken place, the constructive change is necessarily slow, while, if nutrition is seriously impaired, the difficulties of successful management are very considerable. The application of vaccines to patients of this class is quite appropriate and the results are sometimes gratifying. Did opportunity permit I could cite numerous cases under my own observation in which the vaccines proved of appreciable benefit and could present in person a few rather exceptional instances of the remarkable improvement sometimes achieved. Notwithstanding these decidedly picturesque examples, which though impressive are 
infrequently observed, the fact remains that my own clinical experience suggests for vaccine therapy but a limited field of usefulness. As a routine method of treatment in pulmonary tuberculosis it cannot be too strongly condemned. As a special measure of occasional value, but often of doubtful utility, it appears worthy of a judicious trial in selected cases.

Steadman Building.

\section{New Instruments and Suggestions}

\section{A SEMICIRCULAR CANAL INDICATOR}

OF AID IN MAKING THE ROTARY AND CALORIC TESTS OF THE VESTIBULAR APPARATUS

J. W. Downey, JR., M.D., Baltimore

Surgeon, Baltimore Eye, Ear and Throat Hospital; Associate in Otology, University of Maryland, School of Medicine and College of Physicians and Surgeons

After the examination of a large number of patients drawn from the neurologic clinic of Dr. A. C. Gillis at the Miercy Hospital, and from my otologic clinic at the Baltimore Eye, Ear and Throat Hospital, Dr. Gillis and I feel that the vestibular tests of Bárány are worthy of the most elaborate study and that they furnish a wide field for original investigation.

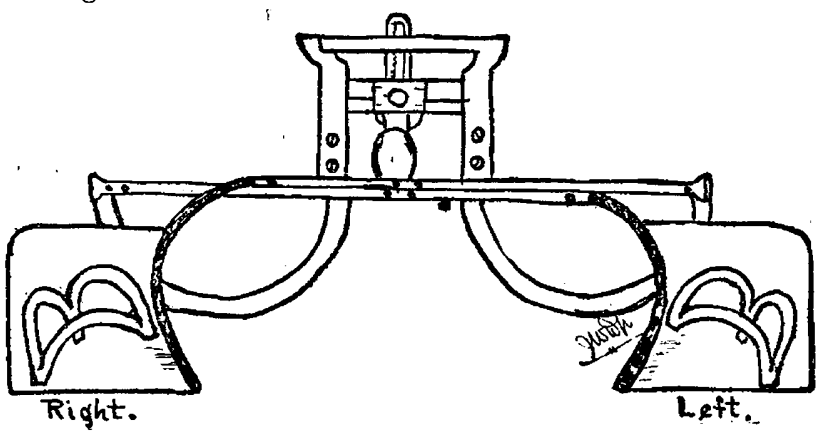

Semicircular canal indicator; the drawing is made from the rear view of the trial frame with the bows folded.

We have assured ourselves that in normal persons the reactions to the rotary and the caloric tests will come through simply and conclusively. Therefore, we believe that al normal reactions are undoubtedly due to some interference in the course of arcs whose complexity have, so far, baffled demonstration.

We accept the postulate of Randall and Jones of Philadclphia, that the fibers from the different semicircular canals have entirely separate tracts of their own, and we have corroborated their findings to our own satisfaction in a series of cases on which we hope later to report. We believe, therefore, that it is of the utmost importance to make the rotary tests with the patient's head in such positions as will bring into horizontal plane the various pairs of canals, for it is only in this plane that stimulation can be aroused by rotation, and the caloric tests with the canals in the vertical position, for similar reasons.

The mental concentration necessary to carry the impression of so many factors is sometimes distracting in the general summing up of our findings. I have, therefore, devised this semicircular canal indicator, which consists of models of the canals mounted on the bows of a trial frame. With the trial frame fitted to the patient, the canals are in approximately the correct position, and a glance will show the ones that are being acted on by the rotation, or the douching, in any position of the head. Strong plus lenses, suggested and used by many observers, the more carefully to study the nystagmus, may be used in the trial frames. A bliclifixator modeled after that of Bárány may also be attached.
I feel that this indicator has the advantage over the Brünings otogonimeter in that it is not in the way in any position it may be desired to place the patient's head, and though, perhaps, not so accurate mathematically, it is vastly superior in demonstrating the position in space of the various canals to those who are not constantly visualizing the anatomic peculiarities of the internal ear.

529 North Charles Street.

\section{CUTANEOUS TUBERCULIN TEST BY PUNCTURE WITH A SOLID NEEDLE}

\section{A. Craig, M.D., London, Ont.}

Superintendent, Queen Alexandra Sanatorium; Chief of Tuberculosis Division, Institute of Ptblic Health, London, Canada

In tuberculosis dispensary work in the examination cf contact cases, the various skin tuberculin tests have proved of considerable value, particularly in children. Some time ago, Dr. H. W. Hill of the Institute of Public Health here began vaccinating against smallpox by means of puncture with a solid needle through a drop of vaccine. His method was successful, and the detail of the procedure has been reported. ${ }^{1}$

The cutaneous tuberculin tests have been extensively used in our city dispensary, and it was decided to try the skin test by puncture through a film of tuberculin. After trying a large number of cases side by side with the von Pirquet test and also a number of definite open cases, we have come to the conclusion that the puncture test has many advantages, and we are using it constantly in our work.

For the test, pure undiluted "O. T." is uged. For the control, we have used glycerinated broth, as it approaches most nearly the basic principle of the tuberculin. For making the puncture a solid needle is used, preferably an ordinary sewing needle. The procedure of the test is as follows:

The skin of the forearm is washed with soap and water, dried, and alcohol or ether is applied and allowed to dry. A thin film of the control solution is placed on the skin, and a series of small punctures made through the film obliquely with a sterilized sewing needle. The punctures should be quite superficial, and in no case should the operator draw blood. Only the extreme tip of the needle is introduced. A film of pure "O. T." is placed on the skin $1 \frac{1 / 2}{2}$ inches below the control area, and punctures are made through this in a similar way. Six punctures in a space one eighth of an inch square are sufficient. A circle may be drawn around the area with a flesh pencil or pen, and each area marked. The test areas may then be entirely wiped off, as it is not necessary to allow the tuberculin or control to dry. The reaction is produced in this test by the slight amount of tuberculin which is carried under the superficial layer of the skin by the point of the needle, and not by the tuberculin on the surface. Reaction, when present, usually occurs in from twelve to forty. eight hours, and is marked by an infiltration of the tuberculiin area. The patient should be instructed not to wash the arm until the test has been observed.

This is in reality a modified intradermal test. In a large number of cases which we have tested, the control entirely disappeared; and when the test is negative, were it not for the marking by the skin pencil or pen, one would be unable to find the test areas. The advantages which this test seems to hold are that the traumatic tissue reaction is extremely slight, and the personal factor of scarification, as in the von Pirquet test, is largely done away with. This is proved by the fact that no evidence of the control can as a rule be found the day following the test. The procedure is painless when properly carried out, and the patient does not have to wait until the film dries, thus saving considerable time. Moreover, the patient cannot rub off the tuberculin or infect the tested areas. In conclusion, one would caution that the tuberculin from the tuberculin area be not transferred to the control area by means of the needle or the absorbent cotton used in wiping off the tuberculin. The same needle or

1. Hill, H. W.: Canad. Med. Assn. Jour., March, 1916. 